\title{
LA RELACIÓN 2D:4D MUESTRA UNA LEVE CORRELACIÓN POSITIVA CON LA DOMINANCIA AGRESIVA, PERO NO CON LA DOMINANCIA SOCIAL, EN JUGADORES DE FÚTBOL DE CATEGORÍAS FORMATIVAS DE UN EQUIPO DE PRIMERA DIVISIÓN EN URUGUAY
}

\section{MILD POSITIVE CORRELATION OF THE 2D:4D RATIO AND AGGRESSIVE DOMINANCE, BUT NOT SOCIABLE DOMINANCE, IN JUNIOR SOCCER PLAYERS FROM AN URUGUAYAN FIRST DIVISION TEAM}

\author{
Álvaro Mailhos \\ Universidad Católica del Uruguay \\ Abraham P. Buunk \\ Universidad de Groningen, Holanda \\ Denise del Arca \\ Universidad Católica del Uruguay
}

\begin{abstract}
Resumen: Existe considerable evidencia que apoya la existencia de una diferencia en los niveles de agresividad entre hombres y mujeres. Se ha propuesto además que los niveles de testosterona (T) estarían relacionados con las conductas agresivas. En este estudio se explora la eventual correlación entre dos formas de dominancia -la dominancia social y la dominancia agresiva- y una medida indirecta de los niveles de $\mathrm{T}$ fetal, la relación 2D:4D, en un grupo de jugadores de fútbol de las categorías formativas de un equipo de Primera División del Uruguay. Los resultados obtenidos muestran una leve correlación positiva de la relación 2D:4D y la dominancia agresiva, pero no con la dominancia social. En este trabajo se discuten los resultados a la luz de observaciones dispares observadas en otras poblaciones y se presentan algunas propiedades psicométricas de las escalas de dominancia social y agresiva empleadas.
\end{abstract}

Palabras Clave: 2D:4D, dominancia social, dominancia agresiva, personalidad, testosterona prenatal

\begin{abstract}
There is considerable evidence supporting the existence of a difference in levels of aggression between men and women. It has also been proposed that testosterone levels could be related to aggressive behavior. This study explores the possible correlation of two different forms of dominance -sociable and aggressive dominance- and an indirect measure of fetal testosterone levels, the 2D:4D ratio, in a group of soccer players from the formative categories of a First Division team in Uruguay. The results obtained show a mild positive correlation of the 2D:4D ratio and aggressive dominance, but not sociable dominance. This paper discusses the results in the light of conflicting observations made in other populations. Additionally, basic psychometric properties of the sociable- and aggressive dominance scales applied in this study are presented.
\end{abstract}

Keywords: 2D:4D, aggressive dominance, sociable dominance, personality, prenatal testosterone

\section{Antecedentes}

Diversos estudios muestran que la agresión física presenta diferencias entre los sexos, observándose en los varones mayores niveles de agresividad física que en las mujeres (Campbell, 2006).

Si bien la caracterización de las conductas agresivas es muy compleja, se ha propuesto que la selección sexual constituiría una mejor explicación a las diferencias en los niveles de agresión intersexual, así como sus causas y variabilidad frente a explicaciones biosociales alternativas (Archer, 2009). Se ha sugerido que las diferencias en los niveles de agresión entre machos y hembras, en mamíferos, podrían resultar de una menor proporción de hembras receptivas en relación a los machos fértiles (Clutton-Brock \& Vincent, 1991).

Adicionalmente, se ha propuesto que la $\mathrm{T}$ desempeñaría un rol importante en la agresión. El dimorfismo sexual observado en los niveles 
de agresión surgiría tempranamente como resultado de los efectos organizadores de la T en las etapas tempranas del desarrollo y se fortalecería al comienzo de la vida adulta, probablemente como resultado de las variaciones en los niveles de esta hormona durante la pubertad (Archer, 2009; Simpson, 2001).

Los conceptos de agresión y dominancia están estrechamente relacionados. Sin embargo, es importante hacer algunas distinciones. Mazur y Booth (1998) proponen que las conductas agresivas son aquellas que están orientadas a infligir un daño físico a otro miembro de la misma especie, en tanto que la dominancia estaría orientada a alcanzar o mantener un estatus elevado. En este sentido, Mazur (1973) plantea que, mientras que los roedores tratan de dominar a otros individuos a través de la agresión, este no sería el principal mecanismo de establecimento de la dominancia entre los primates superiores.

La T estaría relacionada con la competencia y la lucha por alcanzar un estatus dominante, conductas vinculadas con el conflicto y la agresión. Los niveles hormonales podrían predecir la agresión y las conductas asociadas cuando las condiciones favorecen los conflictos interpersonales y la provocación (Geen, 2001).

En los últimos tiempos se han ido acumulando evidencias que apoyan la hipótesis de que la $T$ intrauterina tiene un rol organizador, durante el desarrollo embrionario, de diferentes estructuras anatómicas, incluyendo el sistema nervioso (Bao \& Swaab, 2011; Hines, 2006; Knickmeyer \& Baron-Cohen, 2006). Esta acción organizadora de la T, o de sus metabolitos, puede afectar la supervivencia y la conectividad neuronal y de esa forma provocar consecuencias duraderas. Así la exposición a la T en períodos críticos durante el desarrollo embrionario podría afectar de forma permanente la conducta (Morris, Jordan, \& Breedlove, 2004). En esta misma dirección, Phoenix, Goy, Gerall y Young (1959), en un experimento clásico, expusieron fetos hembra de cobayo a la T y observaron que las conductas copulatorias de estos animales, al alcanzar la madurez sexual, se parecían más a las conductas copulatorias masculinas que a las femeninas.

Por otra parte, se ha propuesto que la relación entre la longitud de los dedos índice y anular (2D:4D) sería un indicador de los niveles de $T$ intrauterina a los que estuvo expuesto un individuo durante su desarrollo temprano (Manning et al., 2000). En tal sentido, se ha observado que relaciones 2D:4D bajas están asociados a altos niveles de $T$ intrauterina (Lutchmaya, Baron-Cohen, Raggatt, Knickmeyer, \& Manning, 2004). Esta relación entre los niveles de T durante el desarrollo fetal y el cociente 2D:4D es también puesta en evidencia por la observación de que la manipulación experimental de los niveles de $T$ fetal resulta en una alteración de las relaciones entre los diferentes dígitos en el faisán común (Romano, Rubolini, Martinelli, Bonisoli Alquati \& Saino, 2005). Si bien esta especie animal se encuentra filogenéticamente lejana al hombre, los mecanismos moleculares de señalización que controlan el desarrollo de los miembros están altamente conservados en vertebrados (Tickle \& Eichele, 1994; Zakany \& Duboule, 2007).

Se ha observado que individuos que sufren de hiperplasia adrenal congénita (HAC) -y por lo tanto están expuestos a niveles elevados de andrógenos adrenales- presentan manos relativamente más masculinizadas. Las mujeres que sufren de HAC presentan cocientes $2 \mathrm{D}: 4 \mathrm{D}$ en la mano izquierda menores que las mujeres que no presentan esta condición, observándose también que los varones con HAC presentan una relación 2D:4D menor que familiares masculinos sanos (Brown, Hines, Fane, \& Breedlove, 2002). Zheng y Cohn (2011) han mostrado que la relación 2D:4D está controlada por el balance entre andrógenos y estrógenos durante una estrecha ventana temporal del desarrollo embrionario, presentando la actividad de los receptores para andrógenos y estrógenos diferencias importantes en los diferentes dígitos.

La relación 2D:4D -y por lo tanto los niveles de T durante el desarrollo embrionario-- ha sido correlacionada con variables fisiológicas, psicológicas y características de la personalidad. Por ejemplo, Manning, Scutt, Wilson y Lewis-Jones, (1998) observaron una correlación positiva entre relaciones 2D:4D altas y bajos conteos espermáticos y niveles reducidos de testosterona en hombres adultos; Bailey y Hurd (2005) observaron que en los hombres la relación 2D:4D correlacionaban positivamente con niveles relativamente elevados de depresión rasgo, mientras que Manning, Baron-Cohen, Wheelwright y Sanders (2001) determinaron que las familias con hijos que presentaban trastornos del espectro autista exhibían relaciones 2D:4D menores que la población general. Por su parte, Luxen y Buunk (2005) reportan que la 
relación 2D:4D de la mano derecha correlaciona positivamente con la amabilidad (agreableness) tanto en hombres como en mujeres; en tanto que estos cocientes correlacionan positivamente con la habilidad verbal y negativamente con la habilidad matemática tanto en hombres como en mujeres. La relación 2D:4D ha sido también correlacionada con la rentabilidad a largo plazo de operadores bursátiles y el tiempo que los mismos permanecen en actividad (Coates, Gurnell, \& Rustichini, 2009). Grimbos, Dawood, Burriss, Zucker y Puts (2010), a través de un meta-análisis, observaron que en las mujeres, la orientación homosexual estaba correlacionada con relaciones 2D:4D más bajas. Manning y Taylor (2001) reportan que hombres con cocientes 2D:4D menores reportan un mejor desempeño en diferentes deportes, y que los jugadores de fútbol profesionales presentan un coeficiente 2D:4D menor que los individuos del grupo control. Estos autores proponen que los niveles de T prenatales y adultos promueven el desarrollo y mantenimiento de características que son útiles en los deportes y en la competencia intrasexual en los hombres.

Diversos estudios han buscado correlacionar los efectos potenciales de los niveles de $T$ prenatal -reflejada en la relación 2D:4D- y los niveles de agresión en diferentes etapas vitales. Un meta análisis de los distintos estudios muestra que la correlación entre el cociente 2D:4D y la agresión no es concluyente en el caso de las mujeres, siendo esta correlación modesta en el caso de los varones (Hönekopp \& Watson, 2011). Sin embargo, las correlaciones pueden estar oscurecidas por haberse utilizado diferentes operacionalizaciones de la agresividad en los distintos estudios. En este sentido, Simpson (2001) sugiere que la T desempeñaría un rol facilitador sólo sobre algunas formas de agresión. Según los autores, se ha sugerido también que la T podría estar relacionada con la motivación de la agresión más que con la violencia propiamente dicha.

Golby y Meggs (2011) analizaron diferentes características psicológicas y su relación con el cociente 2D:4D en una población que incluía deportistas pertenecientes a diferentes disciplinas. Sorprendentemente, estos autores observaron que la relación 2D:4D correlacionaba positivamente con los niveles de hostilidad y negativamente con el nivel de desempeño deportivo alcanzado.
En este estudio exploramos la relación entre los niveles de T fetal -operacionalizados como la relación 2D:4D- la dominancia social y la dominancia agresiva. Estudios similares fueron llevados a cabo por van der Meij, Almela, Buunk, Dubbs y Salvador (2012) utilizando una población de estudiantes universitarios. Este estudio apunta a contribuir al estudio de las variables mencionadas en una muestra por oportunidad conformada por jugadores de fútbol pertenecientes a las categorías formativas de un equipo de Primera División en Uruguay.

\section{Método}

\section{Participantes}

Participaron libremente de este estudio 112 jugadores, varones caucásicos, de las categorías formativas de un club de fútbol de Primera División del Uruguay, con edades comprendidas entre los 13 y los 19 años ( $M=15,81$, $D S=1,55)$. De acuerdo a lo informado por las autoridades del club, ninguno de los jugadores presentaba problemas médicos, psicológicos serios o de abuso de drogas.

\section{Dominancia Social y Dominancia Agresiva}

Los participantes completaron dos cuestionarios para evaluar los niveles de dominancia social y dominancia agresiva (Kalma, Visser, \& Peeters, 1993). Según reportan estos autores, ambas escalas son fiables y fueron validadas a través de la observación de comportamientos no verbales. De acuerdo a la descripción original, la escala de dominancia social y de dominancia agresiva presentaban un Alfa de Cronbach de 0,79 y 0,68 respectivamente. Estos cuestionarios constan de 8 ítems y 7 ítems respectivamente. Esta escala fue traducida al castellano y caracterizada psicométricamente por van der Meij et al. (2012), sin embargo esta traducción presentaba modismos que no se adaptaban a los usos locales de este idioma en el Uruguay. Para su administración, se tradujeron los diferentes ítems a partir de la versión publicada por Kalma et al. (1993). Las traducciones fueron evaluadas y corregidas por dos investigadores con sólida formación en psicología, profundo conocimiento del inglés y hablantes nativos de castellano. Los participantes debieron contestar en qué medida estaban de acuerdo o en desacuerdo con las diferentes 
afirmaciones según una escala iba desde el 1 (totalmente en desacuerdo) hasta el 5 (totalmente de acuerdo). En cuanto a su fiabilidad, para la muestra analizada en este estudio, las escalas de dominancia social y de dominancia agresiva presentan un Alfa de Cronbach de 0,76 y de 0,77 respectivamente.

Los cuestionarios fueron administrados colectivamente en las instalaciones del club de fútbol al que pertenecen los participantes.

\section{Relación 2D:4D}

Las caras ventrales de las manos derechas e izquierdas de los participantes fueron escaneadas usando un escáner CanoScan LiDE 110 Canon. Las imágenes fueron guardadas en formato JPG con un tamaño de 1275 x 1250 píxeles, una resolución de 150 dpi y una profundidad de color de 24 bits por píxel. La luminosidad y contraste de las imágenes fueron ajustados de forma de permitir una mejor visualización de los pliegues de la mano. Las medidas de los dedos índice (2D) y anular (4D) de ambas manos fueron tomadas por dos investigadores independientes con ayuda de la herramienta de medida del software de edición de imágenes GIMP (Versión 2.8). Las medidas de los dedos fueron tomadas desde el punto medio del pliegue más proximal en la base de los dedos, hasta el extremo distal de los mismos. Cuando las medidas tomadas por ambos investigadores diferían en más de tres desvíos típicos, las medidas de estos dedos fueron tomadas nuevamente. Sólo pudieron ser escaneadas las manos de 94 jugadores. Las imágenes correspondientes a las dos manos de un jugador y una de las manos de otros dos jugadores quedaron fuera de foco y por lo tanto fueron eliminadas de este estudio; los datos pertenecientes a otros dos jugadores no fueron considerados en el presente análisis ya que reportaron lesiones en los dedos 2 y 4 . Se calculó la relación 2D:4D para cada mano, de cada participante, realizando el cociente entre longitud del dedo índice y la longitud del dedo anular.

Siguiendo a Weinberg, Scott, Neiswanger y Marazita (2005), se calcularon el error técnico de medida (TEM) y el error técnico de medida relativo (rTEM) para todas las medidas de dedos y coeficientes - menores valores de TEM y rTEM indican mayor precisión en las medidas. En cuanto a las diferencias entre las medidas de los dedos, se encontraron valores de TEM entre 0,48 y 0,61 y de rTEM entre 0,62 y 0,82. El cociente 2D:4D correspondiente a la mano izquierda mostró un valor de TEM de 0,01 y de rTEM de 0,67 ; mientras que para la mano derecha la relación 2D:4D arrojó un TEM de 0,01 y un rTEM de 0,59.

Por otro lado, con el fin de obtener la fiabilidad entre observadores, se calcularon los coeficientes de correlación intraclase $(\mathrm{CCl})$, modelo de efectos mixtos de dos factores, utilizando una definición de acuerdo absoluto. Para todos los dedos y coeficientes se alcanzaron $\mathrm{CCl}$ de entre 0,95 y 0,99 .

\section{Resultados}

En cuanto a las características psicométricas preliminares de las escalas de dominancia social y dominancia agresiva, la medida de adecuación de muestras de Kaiser-Meyer-Olkin $(\mathrm{KMO}=.639)$ y la prueba de esfericidad de Bartlett (Chi-cuadrado=474.609; $\mathrm{gl}=105 ; \mathrm{p}=.000$ ) indican que es posible realizar el Análisis Factorial Exploratorio (AFE). Este se realizó mediante el método de extracción de Componentes Principales, con rotación Varimax y una solución a dos factores rotados. Los factores detectados fueron dominancia social, que explica el 21,79\% de la varianza, y dominancia agresiva, que explica un $19,49 \%$ adicional de la varianza total. Como puede verse en la Tabla 1, el ítem 4 de dominancia social presenta una carga inferior a 0.40 en ambos factores.

Con el fin de determinar si las escalas dominancia social y dominancia agresiva se encontraban relacionadas con el cociente 2D:4D de ambas manos, calculamos el coeficiente de correlación $r$ de Pearson.

En cuanto a la escala dominancia social, no se observan correlaciones estadísticamente significativas con los cocientes 2D:4D de ambas manos ( $p \geq .050)$ (ver Tabla 2).

Como puede verse en la Tabla 3 , se observa una correlación positiva entre la escala dominancia agresiva y el cociente 2D:4D de la mano derecha $(p<.050)$, no observándose una correlación estadísticamente significativa con el cociente 2D4D de la mano izquierda $(p \geq .050)$.

\section{Discusión}

En un estudio reciente, van der Meij et al. (2012) reportan haber observado una correlación negativa significativa entre la relación 
2D:4D y la dominancia agresiva. Los resultados presentados en este estudio difieren de los reportados por estos autores. Existen algunas diferencias metodológicas y particularidades relativas a la muestra que pueden estar dando cuenta de estas discrepancias.

Si bien en la evaluación de la dominancia social y de la dominancia agresiva en ambos estudios se emplearon traducciones al castellano de las escalas desarrolladas por Kalma et al. (1993), van der Meij et al. (2012) utilizan escalas de 9 y 11 ítems respectivamente, mientras que en este estudio se utilizan traducciones de las escalas originales de 8 y 7 ítems respectivamente. Adicionalmente, en este estudio se utilizaron nuevas traducciones de las escalas, adaptadas a las variantes locales del castellano.

El análisis factorial de componentes principales reportado en el presente trabajo muestra que la mayoría de los ítems de las escalas se ajustan bien a la solución de dos factores propuesta originalmente por Kalma et al. (1993), excepto para el ítem 4 de la escala de dominancia social. Sólo el ítem DS4 de la escala de dominancia social (Ciertamente tengo confianza en mí mismo) presenta cargas menores a 0,40 en ambos factores, no ajustándose al modelo propuesto. Estudios adicionales confirmarán si este ítem aporta información de valor o puede ser eliminado. Los resultados de este análisis

Tabla 1

Cargas de los factores de cada item en los dos factores: Dominancia Social (SD) y Dominancia Agresiva (AD)

\begin{tabular}{|c|c|c|c|}
\hline Escala & Ítem & $\begin{array}{l}\text { Dominancia } \\
\text { Social }\end{array}$ & $\begin{array}{l}\text { Dominancia } \\
\text { Agresiva }\end{array}$ \\
\hline DS1 & No tengo problemas en hablar frente a un grupo de personas & .658 & -.043 \\
\hline DS2 & En la escuela me resultaba fácil hablar frente a la clase & .616 & -.060 \\
\hline DS3 & Sin dudas sería un buen líder & .650 & .037 \\
\hline DS4 & Ciertamente tengo confianza en mí mismo & .293 & -.259 \\
\hline DS5 & No me resulta difícil empezar una conversación en un grupo de personas & .788 & .146 \\
\hline DS6 & No soy tímido con personas desconocidas & .762 & -.039 \\
\hline DS7 & Me gusta asumir responsabilidades & .580 & .002 \\
\hline DS8 & La gente me consulta cuando tiene que tomar decisiones & .484 & .064 \\
\hline DA1 & Puedo mirar a cualquiera a los ojos y mentirle sin que se note & .293 & .590 \\
\hline DA2 & Puedo mentir sin que nadie se dé cuenta & .086 & .641 \\
\hline DA3 & Me parece importante salirme con la mía & .012 & .707 \\
\hline DA4 & Me parece importante salirme con la mía aunque esto genere una discusión & .041 & .780 \\
\hline DA5 & Rápidamente me siento agresivo con la gente & -.018 & .546 \\
\hline DA6 & Hago comentarios astutos e hirientes si la gente los merece & -.165 & .625 \\
\hline DA7 & $\begin{array}{l}\text { Prefiero caerle mal a la gente (por ser poco amable) a que me desprecien } \\
\text { (por no alcanzar mis objetivos) }\end{array}$ & -.061 & .563 \\
\hline
\end{tabular}

Tabla 2

Correlación dominancia social y 2D:4D en ambas manos $(n=84)$

\begin{tabular}{lcc}
\hline & 2D:4D Mano derecha & 2D:4D Mano izquierda \\
\cline { 2 - 3 } Coeficiente de correlación de Pearson & $-0,173$ & $-0,040$ \\
Sig. (bilateral) & 0,116 & 0,718 \\
\hline
\end{tabular}

Tabla 3

Correlación dominancia agresiva y 2D:4D en ambas manos ( $n=83$ )

\begin{tabular}{lcc}
\hline & 2D:4D Mano derecha & 2D:4D Mano izquierda \\
\cline { 2 - 3 } Coeficiente de correlación de Pearson & 0,232 & 0,128 \\
Sig. (bilateral) & 0,035 & 0,250 \\
\hline
\end{tabular}


factorial difieren de los presentados por van der Meij et al. (2012); estos autores proponen la eliminación de cuatro ítems, que de acuerdo a nuestro análisis se ajustan bien al modelo de dos factores planteado por los autores originales de las escalas (Kalma et al., 1993).

En el presente estudio no pudimos confirmar la correlación negativa entre la dominancia agresiva y la relación 2D:4D reportada por van der Meij et al. (2012). Más aún, los análisis ponen de relieve una leve correlación positiva entre la relación 2D:4D, contradiciendo los resultados presentados por estos autores. Diferencias en la traducción y puntajes de las escalas utilizados en los estudios de van der Meij y colaboradores y en el presente estudio, así como las características particulares de los grupos estudiados podrían estar dando cuenta de las diferencias observadas.

En primer lugar, las escalas utilizadas en ambos estudios se basan en traducciones diferentes que dan cuenta de las diferentes variantes del castellano empleadas en España y el Río de la Plata. Adicionalmente, van der Meij et al. (2012) utilizaron una escala de 6 puntos que fuerza las decisiones, en tanto que en este trabajo se usó una escala basada en 5 puntos que permite una opción 'neutral'.

Por otra parte, podrían existir diferencias culturales en las poblaciones estudiadas. Posiblemente, las características de las poblaciones de los sujetos que participaron en ambos estudios podrían estar contribuyendo también a las diferencias observadas. Además de eventuales diferencias culturales, la población considerada en nuestro estudio presenta algunas características particulares. El fútbol es un deporte de equipo en el que las conductas agresivas pueden tener consecuencias negativas de gran impacto. Si estas conductas se dirigen a miembros del propio equipo pueden provocar una disminución del rendimiento deportivo, mientras que si están dirigidas hacia jugadores de equipos rivales puede incrementarse el riesgo de sanciones. Los mecanismos de selección y evaluación de los jugadores podrían estar generando una presión selectiva negativa contra aquellos jugadores con altos niveles de conductas agresivas. En este sentido, Golby y Meggs (2011) observaron una correlación positiva entre la relación 2D:4D y niveles de hostilidad en un estudio llevado a cabo con deportistas de diferentes disciplinas, es decir que aquellos individuos que presentaban manos más masculinizadas presentaban menores niveles de hostilidad. En relación a los resultados publicados por van der Meij et al. (2012), Voracek (2013) señala algunos cuestionamientos metodológicos. Según este autor, la correlación negativa significativa entre la relación 2D:4D y la dominancia agresiva observadas por van der Meij et al. (2012) no difiere significativamente de la relación no significativa entre la relación 2D:4D y la dominancia social reportada. De hecho, Hönekopp y Watson (2011) en un estudio meta-analítico, plantean que los reportes publicados en el campo de las relaciones 2D:4D y las conductas vinculadas a la agresión evidenciarían una muy débil relación entre el cociente 2D:4D y los caracteres vinculados a la agresión en los varones, siendo esta correlación inexistente para las mujeres. Estos autores plantean que asumiendo una débil correlación entre la relación 2D:4D y agresión de $r=-0,10$, muestras mayores de 600 participantes deberían emplearse para alcanzar una potencia estadística de 0,80 (Hönekopp y Watson, 2011). En este escenario, los datos que reportamos deberían ser considerados como un estudio preliminar.

El presente estudio presenta algunas limitaciones. Como se mencionara anteriormente, se centra en la exploración de eventuales correlaciones entre los niveles de $T$ fetal -reflejada en la relación 2D:4D - y sus efectos perdurables sobre los niveles de dominancia social y dominancia agresiva. Este estudio fue llevado a cabo con una muestra por oportunidad conformada por 112 varones pertenecientes a las categorías formativas de un club de fútbol del Uruguay. Si bien las particularidades de la muestra hacen difícil la generalización de los resultados a otras poblaciones, consideramos que estas mismas particularidades otorgan interés al presente estudio. En este sentido, los participantes constituyen una muestra altamente competitiva, donde las dimensiones de liderazgo y agresividad tienen un alto impacto. En el futuro será interesante ampliar estos estudios a otros grupos conformados por atletas de ambos sexos y a la población general. Asimismo, planteamos tomar en consideración la heterogeneidad que algunos autores plantean dentro de la adolescencia en cuanto a las variables estudiadas (e.g., Brame, Nagin, \& Tremblay, 2001; Muñoz-Reyes, Gil-Burmann, Fink, \& Turiegano, 2012) profundizando además en la 
relación de las dimensiones social y agresiva de la dominancia con otras operacionalizaciones de las conductas agresivas.

\section{Referencias}

Archer, J. (2009). Does sexual selection explain human sex differences in aggression? Behavioral and Brain Sciences, 32(3/4), 249-311. doi:10.1017/ S0140525X09990951

Bailey, A. A., \& Hurd, P. L. (2005). Depression in men is associated with more feminine finger length ratios. Personality and Individual Differences, 39(4), 829_ 836. doi:10.1016/j.paid.2004.12.017

Bao, A.-M., \& Swaab, D. F. (2011). Sexual differentiation of the human brain: relation to gender identity, sexual orientation and neuropsychiatric disorders. Frontiers in Neuroendocrinology, 32(2), 214-26. doi:10.1016/j. yfrne.2011.02.007

Brame, B., Nagin, D. S., \& Tremblay, R. E. (2001). Developmental trajectories of physical aggression from school entry to late adolescence. Journal of child psychology and psychiatry, and allied disciplines, 42(4), 503-512. Recuperado de http://www.ncbi.nIm. nih.gov/pubmed/11383966

Brown, W. M., Hines, M., Fane, B. A., \& Breedlove, S. M. (2002). Masculinized Finger Length Patterns in Human Males and Females with Congenital Adrenal Hyperplasia. Hormones and Behavior, 42(4), 380386. doi:10.1006/hbeh.2002.1830

Campbell, A. (2006). Sex differences in direct aggression: What are the psychological mediators? Aggression and Violent Behavior, 11(3), 237-264.

Clutton-Brock, T. H., \& Vincent, A. C. J. (1991). Sexual selection and the potential reproductive rates of males and females. Nature, 351, 58-60.

Coates, J. M., Gurnell, M., \& Rustichini, A. (2009). Second-to-fourth digit ratio predicts success among high-frequency financial traders. Proceedings of the National Academy of Sciences of the United States of America, 106(2), 623-8. doi:10.1073/ pnas.0810907106

Geen, R. G. (2001). Human Aggression (2 $2^{\text {nd }}$ ed.) Buckingham, Philadelphia: Open University Press

Golby, J., \& Meggs, J. (2011). Exploring the organizational effect of prenatal testosterone upon the sporting brain. Journal of Sports Science and Medicine, 10, 445-451.

Grimbos, T., Dawood, K., Burriss, R. P., Zucker, K. J., \& Puts, D. a. (2010). Sexual orientation and the second to fourth finger length ratio: A meta-analysis in men and women. Behavioral Neuroscience, 124(2), 278-87. doi:10.1037/a0018764

Hines, M. (2006). Prenatal testosterone and gender-related behaviour. European Journal of Endocrinology, 155 Suppl, S115-21. doi:10.1530/eje.1.02236

Hönekopp, J., \& Watson, S. (2011). Meta-analysis of the relationship between digit-ratio 2D:4D and aggression. Personality and Individual Differences, 51(4), 381386. doi:10.1016/j.paid.2010.05.003

Kalma, A. P., Visser, L., \& Peeters, A. (1993). Sociable and aggressive dominance: Personality differences in leadership style? The Leadership Quarterly, 4(I), 45-64.
Knickmeyer, C. R., \& Baron-Cohen, S. (2006). Fetal testosterone and sex differences. Early Human Development, 82(12), 755-60. doi:10.1016/j. earlhumdev.2006.09.014

Lutchmaya, S., Baron-Cohen, S., Raggatt, P., Knickmeyer, R., \& Manning, J. T. (2004). 2nd to 4th digit ratios, fetal testosterone and estradiol. Early Human Development, 77(1-2), 23-8. doi:10.1016/j.earlhumdev.2003.12.002

Luxen, M. F., \& Buunk, B. P. (2005). Second-to-fourth digit ratio related to Verbal and Numerical Intelligence and the Big Five. Personality and Individual Differences, 39(5), 959-966. doi:10.1016/j.paid.2005.03.016

Manning, J., Barley, L., Walton, J., Lewis-Jones, D., Trivers, R., Singh, D., ... Szwed, A. (2000). The 2nd:4th digit ratio, sexual dimorphism, population differences, and reproductive success. evidence for sexually antagonistic genes? Evolution and Human Behavior, 21(3), 163-183.

Manning, J. T., Baron-Cohen, S., Wheelwright, S., \& Sanders, G. (2001). The 2 nd to 4 th digit ratio and autism. Developmental Medicine and Child Neurology, 43(3), 160-4.

Manning, J. T., Scutt, D., Wilson, J., \& Lewis-Jones, D. I. (1998). The ratio of 2 nd to 4 th digit length: a predictor of sperm numbers and concentrations of testosterone, luteinizing hormone and oestrogen. Human Reproduction, 13(11), 3000-4.

Manning, J. T., \& Taylor, R. P. (2001). Second to fourth digit ratio and male ability in sport: implications for sexual selection in humans. Evolution and Human Behavior, 22(1), 61-69.

Mazur, A. (1973). A Cross-Species Comparison of Status in Small Established Groups. American Sociological Review, 38(5), 513-530.

Mazur, A., \& Booth, A. (1998). Testosterone and dominance in men. Behavioral and Brain Sciences, 21(3), 353-97.

Morris, J. A., Jordan, C. L., \& Breedlove, S. M. (2004). Sexual differentiation of the vertebrate nervous system. Nature Neuroscience, 7(10), 1034-9. doi:10.1038/nn1325

Muñoz-Reyes, J. A., Gil-Burmann, C., Fink, B., \& Turiegano, E. (2012). Physical strength, fighting ability, and aggressiveness in adolescents. American Journal of Human Biology, 24(5), 611-617. doi:10.1002/ ajhb. 22281

Phoenix, C., Goy, R., Gerall, A., \& Young, W. (1959). Organizing action of prenatally administered testosterone propionate on the tissues mediating mating behavior in the female guinea pig. Endocrinology, 65, 369-82.

Romano, M., Rubolini, D., Martinelli, R., Bonisoli Alquati, A., \& Saino, N. (2005). Experimental manipulation of yolk testosterone affects digit length ratios in the ring-necked pheasant (Phasianus colchicus). Hormones and Behavior, 48(3), 342-6. doi:10.1016/j. yhbeh.2005.03.007

Simpson, K. (2001). The Role of Testosterone in Aggression. McGill Journal of Medicine, 6(1), 32-40.

Tickle, C., \& Eichele, G. (1994). Vertebrate limb development. Annual Review of Cell Biology, 10, 121-52. doi:10.1146/ annurev.cb.10.110194.001005

van der Meij, L., Almela, M., Buunk, A. P., Dubbs, S., \& Salvador, A. (2012). 2D:4D in men is related to aggressive dominance but not to sociable dominance. Aggressive Behavior, 38(3), 208-12. doi:10.1002/ab.21422 
aVoracek, M. (2013). Differential Correlations of Digit Ratio (2D:4D) with Aggressive Dominance and Sociable Dominance Are Not Demonstrated: Commentary on van der Meij, Almela, Buunk, Dubbs, and Salvador (2012, Aggressive Behavior, 38(3), 208-212). Aggressive Behavior, 39(2), 85-7. doi:10.1002/ ab.21463

Weinberg, S. M., Scott, N. M., Neiswanger, K., \& Marazita, M. L. (2005). Intraobserver error associated with measurements of the hand. American Journal of Human Biology, 17(3), 368-371. doi:10.1002/ ajhb.20129
Zakany, J., \& Duboule, D. (2007). The role of Hox genes during vertebrate limb development. Current Opinion in Genetics \& Development, 17(4), 359-66. doi:10.1016/j.gde.2007.05.011

Zheng, Z., \& Cohn, M. J. (2011). Developmental basis of sexually dimorphic digit ratios. Proceedings of the National Academy of Sciences of the United States of America, 108(39), 16289-16294. doi:10.1073/ pnas. 1108312108

Para citar este artículo:

Mailhos, A., Buunk., A., y del Arca, D. (2013). La relación 2D:4D muestra una leve correlación positiva con la dominancia agresiva, pero no con la dominacia social, en jugadores de fútbol de categorías formativas de un equipo de Primera División en Uruguay. Ciencias Psicológicas VII (2): 143 - 150. 